\title{
BMJ Open Effects of number and gender of offspring on quality of life among older adults: evidence from the Korean Longitudinal Study of Aging, 2006-2012
}

\author{
Jae-Hyun Kim, ${ }^{1,2}$ Sang Gyu Lee, ${ }^{1,2}$ Jaeyong Shin, ${ }^{1,2}$ Kyung-Hee Cho, ${ }^{1,2}$ \\ Jae-Woo Choi, ${ }^{1,2}$ Eun-Cheol Park ${ }^{2,3}$
}

To cite: Kim J-H, Lee SG, Shin J, et al. Effects of number and gender of offspring on quality of life among older adults: evidence from the Korean Longitudinal Study of Aging, 2006-2012. BMJ Open 2015;5:e007346. doi:10.1136/bmjopen-2014007346

- Prepublication history for this paper is available online. To view these files please visit the journal online (http://dx.doi.org/10.1136/ bmjopen-2014-007346).

Received 3 December 2014 Revised 12 May 2015 Accepted 13 May 2015

CrossMark

\footnotetext{
${ }^{1}$ Department of Public Health, Graduate School, Yonsei University, Seoul, Republic of Korea

${ }^{2}$ Institute of Health Services Research, Yonsei University, Seoul, Republic of Korea

${ }^{3}$ Department of Preventive Medicine, Yonsei University College of Medicine, Seoul, Republic of Korea
}

Correspondence to Dr Eun-Cheol Park; ecpark@yuhs.ac

\section{ABSTRACT}

Objectives: We examined correlations between number and gender of offspring and health-related quality of life (HRQoL) and quality of life $(\mathrm{Q} o \mathrm{~L})$ in older adults.

Setting: We used data from the 2006-2012 data sets of the Korean Longitudinal Study of Aging.

Participants: There were $10242,8680,7907$ and 7480 participants in 2006, 2008, 2010 and 2012, respectively.

Interventions: Number and gender of offspring. Primary and secondary outcome measures: We measured participants' QoL and HRQoL using a visual analogue scale developed by the Korea Labour Institute and which is similar to the EQ-VAS, a European measure.

Results: We estimated the HRQOL and QoL of individuals with offspring. Estimates for the HRQoL and $\mathrm{QoL}$ of parents with no offspring were -7.762 and -9.384 , respectively (both $p<0.0001$ ) versus parents with two offspring. For parents with five or more offspring, the estimates for the HRQoL and QoL were -1.529 and 0.885 , respectively $(p<0.001$ and $p<0.017$, respectively) compared with parents with two offspring. For fathers with no offspring compared with fathers with two offspring, the estimates for the HRQoL and QoL were -6.143 and -7.492 , respectively (both $p<0.0001$ ).

Conclusions: These results suggest that number of offspring is associated with both $\mathrm{HRQOL}$ and QoL. Those with no offspring showed the lowest $\mathrm{HRQ} \mathrm{LL}$ and QoL. Although having five or more children had positive associations with QoL, it had negative associations with HRQoL. Public health services for those with poor quality of life should provide effective support programmes and services based on these findings.

\section{INTRODUCTION}

Although the Korean population has been increasing steadily, reaching 47.99 million in 2010, the fertility rate between 2005 and 2010
Strengths and limitations of this study

- This study used nationwide longitudinal survey data of community-dwelling adults; the large population sample size was representative of the overall population, so these results can be generalised to the population of adults $\geq 45$ years of age in South Korea.

- Respondent reports are subjective and imperfect measures, potentially affected by perception bias and adaptation of resources.

- We recognise that the estimates may have understated the potential associations for all of the outcomes because we used a short follow-up period in our analysis.

- Twins or triplets might have been present in the population, but we did not examine composition of offspring because of insufficient sample sizes, nor could we determine whether adults with no offspring were childless as a result of infertility.

- We used longitudinal data for our estimates, but the results possibly reflected reverse causality and bidirectional relationships when the associations between number of offspring and quality of life were assessed.

was the lowest in the world. The number of live births declined from 1006654 in 1970 to a low of 435031 in 2005 , then slightly increased to 470171 in 2010. ${ }^{1}$ In June 2006, the Presidential Committee on the Aging Society and Population Policy introduced a basic plan for a low-fertility and aging society. The first basic plan (2006-2010) aimed to foster environments favouring child-rearing and to establish a base for improving quality of life in an aging society. The second basic plan (20112015) aimed to steadily increase fertility to previous rates, and to consolidate social supports for the elderly. The third plan (2016-2020) aims to raise the fertility rate to the average level in OECD countries, and to encourage society to adapt for an aging population. ${ }^{1}$ 
Another problem is the rapid increase in the elderly population. Populations worldwide have been rapidly aging since 2000, and particularly in South Korea. The proportion of the population considered to be aged increased from $3.8 \%$ in 1980 to $11 \%$ in 2010 . Estimates indicate that the proportion of older adults will increase geometrically, to $24.3 \%$ in 2030 and $37.4 \%$ in $2050 .^{2}$ Accordingly, the requirements of this population have become an important public health issue.

The increase in life expectancy may be an indicator of the quality of the state welfare systems in South Korea. However, a longer lifespan does not always indicate quality survival for older adults. Thus, because of this increased longevity and life expectancy, quality of life (QoL) has become an important issue in the public health sector. ${ }^{3}$

According to the WHO Quality of Life Group, 'Quality of life is the individual's perception of their position in life in the context of the culture and the value systems in which they live in relation to their goals, expectations, standards, and concerns. It is a broad concept affected in a complex way in which the person's physical health, psychological state, level of independence, and social relationships are salient features of their environment'. ${ }^{4}$

Health, functional status, social support (especially the support of family and friends) and social relationships are among the main issues that affect the QoL of older adults. ${ }^{5}$ Robinson and Molzahn ${ }^{6}$ stated that personal relationships and health status are the most important factors that explain differences in the QoL of older people. Social support and variables pertaining to social networks (family, children and friends) are also important factors related to the QoL of older adults. For the subscales of sensory abilities and death-and-dying, older adults with offspring have higher mean QoL variable scores than adults without offspring. Notwithstanding the arguments for and against having children and the effects on mental health, many studies have shown that offspring make important contributions to the social network of aging parents. ${ }^{7}$ Offspring can provide social support and care to prevent loneliness in old age. Loneliness was a significant factor for older people living alone with lower QoL. ${ }^{8}$ An extensive literature has noted the adverse impact of loneliness upon physical and psychological health, which may eventually impair QoL. ${ }^{10}$ Having offspring also causes parents to feel grateful and gives meaning to their life, which may positively affect mental health. ${ }^{11}$

QoL is used to describe the general well-being of a population, which is a subjective and complex concept. ${ }^{12}$ Health-related quality of life (HRQoL) indicates how an individual physically feels about him/ herself, and the extent to which they are capable of fulfilling their daily responsibilities and achieving their aims. In the psychological sense, HRQoL assesses the ability to feel and express emotions such as anger, resentment, fear and happiness. ${ }^{13}$ Thus, the concept of HRQoL is often used to measure the effect of illness on
QoL. ${ }^{14}$ The objective of our study was to investigate whether offspring reduce or increase HRQoL and QoL.

\section{METHODS}

\section{Study sample and design}

Data were from the 2006-2012 data sets of the Korean Longitudinal Study of Aging (KLoSA), a nationwide survey of community-dwelling adults aged $\geq 45$ years. A multistage stratified cluster sampling design was used for the KLoSA study, and data collection was repeated every even-numbered year. The information was collected by the Korea Labour Institute and consisted of the basic data needed to devise and implement effective social and economic policies to address emerging trends related to population aging.

The original KLoSA study population consisted of South Korean adults aged $\geq 45$ years who lived in 15 large administrative areas (Seoul, Incheon, Kyunggi, Kangwon, Busan, Ulsan, Kyungnam, Daegu, Kyungbuk, Kwangju, Chonbuk, Chunnam, Daejeon, Chungbuk and Chungnam). KLoSA maintains a national public database (http://www.kli.re.kr/klosa/en/about/introduce.jsp). ${ }^{15}$

Our study used samples from the first- to fourth-wave KLoSA data sets. In the first baseline survey in 2006, 10254 individuals in 6171 households (1.7 individuals per household) were interviewed using a computerassisted personal interviewing method. The second survey, in 2008, followed up 8688 subjects, representing $86.6 \%$ of the original panel. The third survey, in 2010 , followed up 7920 subjects, representing $80.3 \%$ of the original panel. The fourth survey, in 2012, followed up 7486 subjects, representing $76.2 \%$ of the original panel.

We excluded 12 of the 2006 survey participants as they lacked information ( 5 had missing data on education, 3 on income, 2 on smoking status and 2 on chronic disease). We excluded 8 subjects with missing information in 2008 (5 lacked data on QoL, 2 on education and 1 on smoking status), 13 subjects with missing information in 2010 (10 lacked data on QoL, 1 on education and 2 on chronic disease), and 6 subjects with missing information in 2012 ( 1 lacked data on education, 1 on smoking status and 4 on chronic disease).

\section{Offspring-related independent variables}

The number of offspring and composition of offspring were the independent variables of interest. The average age of offspring was divided into four categories: Q1 ( $\leq 27.5$ years), Q2 (27.6-36.0 years), Q3 (36.1-44.0 years) and Q4 ( $\geq 44.0$ years).

\section{Control variables}

We divided the population into seven age groups at 5-year intervals: $\leq 49,50-54,55-59,60-64,65-69,70-74$ and $\geq 75$ years. Education status was divided into five categories: no schooling, elementary school, middle school, high school and college or above. Income status was divided into two categories: Yes or No. The frequency of 
visiting friends was divided into five categories: every day, 1-2 times per week, 1-2 times per month, 3-6 times per year, and never. Economic activity status was divided into two categories: employed and unemployed. Self-rated health characteristics were also included as covariates in our analyses. In response to the question: 'Have you felt sadness or despair that hindered everyday life consistently for 2 weeks or more during the last year?', depressive symptoms were subcategorised as 'yes' or 'no'. Smoking status and alcohol use were divided into three categories: yes, former user or no.

\section{Dependent variables}

\section{HRQOL}

HRQoL was measured as a response to the simple question: ${ }^{16} 17$ 'How do you usually perceive your health-related quality of life?'. Although the measure was developed by the Korea Labour Institute, it is similar to EQ-VAS, which also consists of only one question: 'How do you usually perceive your health-related quality of life?'. The scale ranges from 0 (worst health state) to 100 (best health state) points.

\section{Overall subjective QoL}

Subjective QoL was measured with a simple question: 'How is your overall quality of life?' and was a proxy indicator of the current health status of respondents indicated on a vertical visual analogue scale from zero to 100 , marked in tens. The endpoints were labelled 'best imaginable overall state' and 'worst imaginable overall state', ranging from 0 (worst overall state) to 100 (best overall state).

\section{Analytical approach and statistics}

Analysis of variance and mixed modelling were used to investigate the effects of offspring on quality of life (HRQoL, QoL) among older adults. For all analyses, a $\mathrm{p}$ value $\leq 0.05$ (two-tailed test) was considered statistically significant. All analyses were performed using SAS software V.9.2 (SAS Institute, Cary, North Carolina, USA).

\section{Mixed-effects model (SAS Proc Mixed)}

A mixed model was used to handle unbalanced data with correlated outcomes and missing data. In all mixed models, only the intercept was allowed to vary between subjects, and the regression slopes were assumed to be fixed effects; random intercept models were applied to the data. The random intercept variance is reported as $\sigma^{2}{ }^{18}$

The outcome in mixed models was QoL as a continuous variable. Covariates of interest from all subjects were added to the model to determine their effects on the probability of reporting changed QoL. To determine whether the probability of changed QoL varied over time, we included time (year) in the model as a categorical covariate; the regression coefficient was used to estimate both the change in probability of altered QoL and independent variables annually. ${ }^{18}$

\section{RESULTS}

Table 1 presents the general characteristics at baseline of the covariates included in the study and of additional variables of interest (eg, marital status and average number of offspring). Data from 10242 individuals were included at baseline.

For the number of offspring at baseline, the weighted mean for the QoL and HRQoL of those with no offspring was 46.0 and 46.4 , respectively (father mean: 46.5 and 48.6; mother mean: 45.5 and 43.4; table 1 ). The weighted mean for the QoL and HRQoL of those with one offspring was 60.4 and 58.9, respectively (father mean: 61.3 and 61.6; mother mean: 59.6 and 56.5). The weighted mean for the QoL and HRQoL of those with five or more offspring was 58.5 and 47.1, respectively (father mean: 60.4 and 52.8; mother mean: 57.5 and 44.3; table 1).

Tables 2 and 3 and figures 1 and 2 show the estimates derived using a mixed-effects model. Table 2 presents the results for the adjusted effects of number of offspring on HRQoL and QoL. For those with no offspring, the estimates for HRQoL and QoL were -7.762 and -9.384, respectively (SE: 0.679 and 0.606 ; both $\mathrm{p}<0.0001$ ) compared with those with two offspring. For those with five or more offspring, the estimates for HRQoL and QoL were -1.529 and 0.885 , respectively (SE: 0.414 and 0.369 ; $\mathrm{p}<0.001$ and $\mathrm{p}<0.017)$ compared with those with two offspring. For fathers with five or more offspring compared with fathers with two offspring, the estimates for HRQoL and QoL were -6.143 and -7.492 (SE: 0.949 and 0.829; both $\mathrm{p}<0.0001$ ), respectively. For fathers with five or more offspring, the estimates for HRQoL and QoL were -0.400 and 1.304, respectively. For mothers with no offspring, the estimates for HRQoL and QoL were -8.372 and -8.998 , respectively (SE: 1.031 and 0.934 ; both $\mathrm{p}<0.0001)$ compared with mothers with two offspring. For mothers with five or more offspring, the estimates for HRQoL and QoL were -1.897 and 0.797 , respectively (SE: 0.516 and $0.467 ; \mathrm{p}<0.001$ and $\mathrm{p}<0.090$ ) compared with mothers with two offspring.

Tables 4 and 5 present results for the adjusted effect of the composition of offspring on HRQoL and QoL. For those with no boys and no girls (no offspring), the estimates for HRQoL and QoL were -7.943 and -9.377 , respectively (SE: 0.697 and 0.621 ; both $\mathrm{p}<0.0001$ ) compared with those with one boy and one girl. For those with two or more boys and two or more girls, the estimates for HRQoL and QoL were 1.537 and -0.058 , respectively (SE: 0.422 and $0.377 ; \mathrm{p}<0.001$ and $\mathrm{p}<0.878$ ) compared with those with one boy and one girl.

For fathers with no boys and no girls, the estimates for HRQoL and QoL were -6.410 and -7.356 , respectively (SE: 0.975 and 0.852 ; both $\mathrm{p}<0.0001$ ) compared with fathers with one boy and one girl. For fathers with two or more boys and two or more girls, the estimates for HRQoL and QoL were -1.575 and 0.405 , respectively (SE: 0.689 and $0.602 ; \mathrm{p}=0.024$ and $\mathrm{p}=0.502$ ) compared with fathers with one boy and one girl. 
Mother

Weighted QoL HRQoL Heighted QoL HoL $\quad$ Weighted QoL

\begin{tabular}{|c|c|c|c|c|c|c|c|c|c|c|c|c|c|c|c|c|c|c|}
\hline & $\mathbf{N}$ & $\%$ & (mean) & p Value & (mean) & p Value & $\mathbf{N}$ & $\%$ & (mean) & p Value & (mean) & p Value & $\mathbf{N}$ & $\%$ & (mean) & p Value & (mean) & p Value \\
\hline Age (years) & & & & $<0.0001$ & & $<0.0001$ & & & & $<0.0001$ & & $<0.0001$ & & & & $<0.0001$ & & $<0.0001$ \\
\hline$\leq 49$ & 1480 & 9.6 & 65.8 & & 64.5 & & 529 & 32.6 & 65.1 & & 65.5 & & 951 & 67.4 & 66.4 & & 63.5 & \\
\hline $50-54$ & 1173 & 8.3 & 64.2 & & 62.7 & & 533 & 44.3 & 65.3 & & 65.1 & & 640 & 55.7 & 63.0 & & 60.2 & \\
\hline $55-59$ & 1508 & 10.8 & 62.4 & & 59.2 & & 675 & 43.4 & 63.1 & & 62.3 & & 833 & 56.6 & 61.7 & & 56.3 & \\
\hline $60-64$ & 1383 & 13.2 & 62.6 & & 56.5 & & 633 & 48.4 & 64.2 & & 61.0 & & 750 & 51.6 & 61.2 & & 52.1 & \\
\hline $65-69$ & 1407 & 13.5 & 58.5 & & 50.7 & & 643 & 49.2 & 61.5 & & 56.3 & & 764 & 50.9 & 56.3 & & 46.5 & \\
\hline $70-74$ & 1505 & 20.4 & 56.8 & & 48.2 & & 668 & 50.7 & 58.1 & & 53.2 & & 837 & 49.3 & 55.7 & & 44.3 & \\
\hline$\geq 75$ & 1786 & 24.2 & 54.4 & & 43.7 & & 776 & 50.4 & 56.0 & & 47.0 & & 1010 & 49.7 & 53.7 & & 42.1 & \\
\hline Gender & & & & $<0.0001$ & & $<0.0001$ & NA & & & & & & & & & & & \\
\hline Male (father) & 4457 & 47.1 & 63.2 & & 61.3 & & & & & & & & & & & & & \\
\hline Female (mother) & 5785 & 53.0 & 60.8 & & 54.2 & & & & & & & & & & & & & \\
\hline Education & & & & $<0.0001$ & & $<0.0001$ & & & & $<0.0001$ & & $<0.0001$ & & & & $<0.0001$ & & $<0.0001$ \\
\hline $\begin{array}{l}\text { Elementary school or } \\
\text { less }\end{array}$ & 4826 & 39.9 & 55.9 & & 48.3 & & 1420 & 30.0 & 56.3 & & 51.7 & & 3406 & 70.0 & 55.8 & & 46.8 & \\
\hline Middle school & 1654 & 16.8 & 61.9 & & 58.6 & & 762 & 47.9 & 60.7 & & 58.4 & & 892 & 52.1 & 63.1 & & 58.7 & \\
\hline High school & 2706 & 30.8 & 66.1 & & 64.1 & & 1497 & 57.0 & 65.2 & & 64.6 & & 1209 & 43.0 & 67.3 & & 63.5 & \\
\hline College or above & 1056 & 12.5 & 71.0 & & 69.4 & & 778 & 75.7 & 70.5 & & 69.5 & & 278 & 24.3 & 72.6 & & 69.0 & \\
\hline Marital status & & & & $<0.0001$ & & $<0.0001$ & & & & $<0.0001$ & & $<0.0001$ & & & & $<0.0001$ & & $<0.0001$ \\
\hline Married & 7959 & 81.0 & 64.4 & & 60.2 & & 4090 & 53.4 & 64.8 & & 62.5 & & 3869 & 46.7 & 63.9 & & 57.6 & \\
\hline $\begin{array}{l}\text { Single (including } \\
\text { separated, divorced) }\end{array}$ & 2283 & 19.0 & 51.6 & & 46.1 & & 367 & 20.2 & 45.6 & & 47.4 & & 1916 & 79.8 & 53.1 & & 45.8 & \\
\hline Income & & & & $<0.0001$ & & $<0.0001$ & & & & $<0.0001$ & & $<0.0001$ & & & & 0.6383 & & $<0.0001$ \\
\hline Yes & 1983 & 23.6 & 65.6 & & 66.0 & & 1334 & 71.0 & 66.8 & & 67.7 & & 649 & 29.0 & 62.6 & & 61.8 & \\
\hline No & 8259 & 76.4 & 60.8 & & 54.9 & & 3123 & 39.7 & 61.3 & & 57.7 & & 5136 & 60.4 & 60.5 & & 53.1 & \\
\hline Economic activity & & & & $<0.0001$ & & $<0.0001$ & & & & $<0.0001$ & & $<0.0001$ & & & & 0.0005 & & $<0.0001$ \\
\hline Yes & 3880 & 45.6 & 65.9 & & 65.0 & & 2490 & 67.6 & 66.9 & & 67.0 & & 1390 & 32.4 & 63.7 & & 60.6 & \\
\hline No & 6362 & 54.4 & 58.7 & & 51.3 & & 1967 & 29.8 & 56.4 & & 50.3 & & 4395 & 70.2 & 59.7 & & 51.7 & \\
\hline $\begin{array}{l}\text { Number of visits with } \\
\text { friends }\end{array}$ & & & & $<0.0001$ & & $<0.0001$ & & & & $<0.0001$ & & $<0.0001$ & & & & $<0.0001$ & & $<0.0001$ \\
\hline Never & 1222 & 12.1 & 53.9 & & 49.9 & & 546 & 48.8 & 53.7 & & 51.7 & & 676 & 51.2 & 54.1 & & 48.2 & \\
\hline $3-6$ times a year & 603 & 6.1 & 58.6 & & 55.8 & & 281 & 49.9 & 60.0 & & 58.5 & & 322 & 50.1 & 57.1 & & 53.1 & \\
\hline $1-2$ times a month & 1828 & 18.9 & 61.6 & & 58.9 & & 941 & 55.8 & 62.9 & & 62.5 & & 887 & 44.2 & 60.0 & & 54.3 & \\
\hline $1-2$ times a week & 3283 & 32.1 & 63.7 & & 59.3 & & 1406 & 46.8 & 65.6 & & 62.9 & & 1877 & 53.2 & 62.0 & & 56.2 & \\
\hline Every day & 3306 & 30.8 & 64.2 & & 58.1 & & 1283 & 40.7 & 66.0 & & 63.4 & & 2023 & 59.3 & 62.9 & & 54.5 & \\
\hline Smoking status & & & & $<0.0001$ & & $<0.0001$ & & & & $<0.0001$ & & $<0.0001$ & & & & $<0.0001$ & & 0.0005 \\
\hline Never & 7291 & 68.7 & 62.5 & & 56.7 & & 1733 & 25.7 & 65.9 & & 62.9 & & 5558 & 74.3 & 61.3 & & 54.6 & \\
\hline Former smoker & 978 & 9.3 & 62.4 & & 57.6 & & 942 & 97.0 & 62.7 & & 58.3 & & 36 & 3.0 & 51.2 & & 33.3 & \\
\hline Smoker & 1973 & 22.1 & 60.1 & & 60.0 & & 1782 & 92.6 & 61.2 & & 61.2 & & 191 & 7.4 & 47.4 & & 45.5 & \\
\hline Alcohol use & & & & $<0.0001$ & & $<0.0001$ & & & & $<0.0001$ & & $<0.0001$ & & & & 0.0004 & & $<0.0001$ \\
\hline Yes & 3883 & 42.5 & 63.7 & & 62.1 & & 2811 & 74.4 & 64.0 & & 63.4 & & 1072 & 25.7 & 62.9 & & 58.1 & \\
\hline Former user & 689 & 6.1 & 55.3 & & 47.5 & & 552 & 79.3 & 56.5 & & 49.6 & & 137 & 20.7 & 50.7 & & 39.5 & \\
\hline No & 5670 & 51.3 & 61.3 & & 55.0 & & 1094 & 20.6 & 64.0 & & 60.1 & & 4576 & 79.4 & 60.6 & & 53.6 & \\
\hline Depressive symptoms & & & & $<0.0001$ & & $<0.0001$ & & & & $<0.0001$ & & $<0.0001$ & & & & $<0.0001$ & & $<0.0001$ \\
\hline Yes & 1225 & 10.9 & 48.3 & & 39.6 & & 363 & 32.3 & 46.7 & & 41.7 & & 862 & 67.8 & 49.0 & & 38.7 & \\
\hline No & 9017 & 89.1 & 63.6 & & 59.7 & & 4094 & 48.9 & 64.6 & & 62.8 & & 4923 & 51.2 & 62.7 & & 56.7 & \\
\hline
\end{tabular}




\begin{tabular}{|c|c|c|c|c|c|c|c|c|c|c|c|c|c|c|c|c|c|c|}
\hline & \multicolumn{6}{|l|}{ Total } & \multicolumn{6}{|c|}{ Father } & \multicolumn{6}{|c|}{ Mother } \\
\hline & $\mathbf{N}$ & $\begin{array}{l}\text { Weighted } \\
\%\end{array}$ & $\begin{array}{l}\text { QoL } \\
\text { (mean) }\end{array}$ & p Value & $\begin{array}{l}\text { HRQoL } \\
\text { (mean) }\end{array}$ & p Value & $\mathbf{N}$ & $\begin{array}{l}\text { Weighted } \\
\%\end{array}$ & $\begin{array}{l}\text { QoL } \\
\text { (mean) }\end{array}$ & p Value & $\begin{array}{l}\text { HRQoL } \\
\text { (mean) }\end{array}$ & p Value & $\mathbf{N}$ & $\begin{array}{l}\text { Weighted } \\
\%\end{array}$ & $\begin{array}{l}\text { QoL } \\
\text { (mean) }\end{array}$ & p Value & $\begin{array}{l}\text { HRQoL } \\
\text { (mean) }\end{array}$ & p Value \\
\hline $\begin{array}{l}\text { Number of chronic } \\
\text { diseases }\end{array}$ & & & & $<0.0001$ & & $<0.0001$ & & & & 0.0033 & & $<0.0001$ & & & & $<0.0001$ & & $<0.0001$ \\
\hline 0 & 5376 & 57.7 & 64.8 & & 64.3 & & 2568 & 51.3 & 65.1 & & 66.3 & & 2808 & 48.7 & 64.5 & & 62.1 & \\
\hline 1 & 2962 & 26.5 & 60.2 & & 52.9 & & 1217 & 43.5 & 62.0 & & 57.3 & & 1745 & 56.5 & 58.8 & & 49.5 & \\
\hline$\geq 2$ & 1904 & 15.8 & 54.4 & & 40.7 & & 672 & 37.4 & 56.4 & & 43.7 & & 1232 & 62.6 & 53.2 & & 38.8 & \\
\hline Number of offspring & & & $<0.0001$ & & $<0.0001$ & & & & $<0.0001$ & & $<0.0001$ & & & & $<0.0001$ & & $<0.0001$ & \\
\hline 0 & 319 & 3.6 & 46.0 & & 46.4 & & 154 & 57.4 & 46.5 & & 48.6 & & 165 & 42.6 & 45.2 & & 43.4 & \\
\hline 1 & 794 & 8.8 & 60.4 & & 58.9 & & 336 & 48.2 & 61.3 & & 61.6 & & 458 & 51.9 & 59.6 & & 56.5 & \\
\hline 2 & 3509 & 40.5 & 64.8 & & 62.5 & & 1691 & 52.8 & 65.5 & & 65.0 & & 1818 & 47.2 & 64.0 & & 59.7 & \\
\hline 3 & 2540 & 23.4 & 63.5 & & 58.5 & & 1130 & 46.1 & 65.0 & & 61.8 & & 1410 & 53.9 & 62.2 & & 55.7 & \\
\hline 4 & 1458 & 11.6 & 58.6 & & 51.4 & & 578 & 39.0 & 60.4 & & 55.3 & & 880 & 61.1 & 57.5 & & 48.9 & \\
\hline$\geq 5$ & 1622 & 12.1 & 58.5 & & 47.1 & & 568 & 33.6 & 60.4 & & 52.8 & & 1054 & 66.4 & 57.5 & & 44.3 & \\
\hline Composition of offspring & & & & 0.0441 & & 0.1313 & & & & 0.0316 & & 0.0275 & & & & 0.6297 & & 0.4036 \\
\hline 0 Boy and 0 girl & 319 & 3.6 & 46.0 & & 46.4 & & 154 & 57.4 & 46.5 & & 48.6 & & 165 & 42.6 & 45.2 & & 43.4 & \\
\hline 0 Boy and 1 girl & 291 & 3.1 & 60.2 & & 58.0 & & 128 & 48.2 & 62.6 & & 62.6 & & 163 & 51.8 & 58.0 & & 53.7 & \\
\hline 1 Boy and 0 girl & 503 & 5.7 & 60.5 & & 59.5 & & 208 & 48.1 & 60.6 & & 61.1 & & 295 & 51.9 & 60.5 & & 58.0 & \\
\hline 0 Boy and $\geq 2$ girls & 707 & 7.8 & 64.0 & & 60.3 & & 332 & 51.5 & 65.9 & & 62.8 & & 375 & 48.5 & 62.0 & & 57.5 & \\
\hline 1 Boy and 1 girl & 1837 & 21.3 & 64.5 & & 62.9 & & 889 & 52.9 & 64.8 & & 65.7 & & 948 & 47.1 & 64.2 & & 59.8 & \\
\hline$\geq 2$ Boys and 0 girl & 1640 & 17.3 & 64.3 & & 60.4 & & 759 & 50.2 & 65.5 & & 63.2 & & 881 & 49.9 & 63.0 & & 57.6 & \\
\hline$\geq 2$ Boys and 1 girl & 1519 & 12.7 & 61.0 & & 54.6 & & 626 & 41.5 & 63.1 & & 58.5 & & 893 & 58.5 & 59.5 & & 51.9 & \\
\hline 1 Boy and $\geq 2$ girls & 1710 & 15.5 & 63.4 & & 57.8 & & 723 & 44.3 & 65.1 & & 62.4 & & 987 & 55.7 & 62.1 & & 54.1 & \\
\hline$\geq 2$ Boys and $\geq 2$ girls & 1716 & 12.9 & 58.1 & & 47.8 & & 638 & 35.7 & 59.4 & & 52.2 & & 1078 & 64.3 & 57.3 & & 45.4 & \\
\hline Number of boys in family & & & & 0.0345 & & $<0.0001$ & & & & 0.2908 & & 0.0003 & & & & 0.0096 & & 0.0068 \\
\hline 0 & 1317 & 14.5 & 58.8 & & 56.4 & & 614 & 52.3 & 60.0 & & 58.9 & & 703 & 47.8 & 57.4 & & 53.6 & \\
\hline 1 & 4050 & 42.6 & 63.6 & & 60.6 & & 1820 & 49.1 & 64.4 & & 64.0 & & 2230 & 50.9 & 62.8 & & 57.3 & \\
\hline$\geq 2$ & 4875 & 43.0 & 61.4 & & 54.9 & & 2023 & 43.2 & 63.3 & & 59.1 & & 2852 & 56.8 & 60.0 & & 51.7 & \\
\hline Number of girls in family & & & & 0.0147 & & $<0.0001$ & & & & 0.2067 & & 0.6872 & & & & 0.0972 & & 0.0856 \\
\hline 0 & 2462 & 26.6 & 61.0 & & 58.3 & & 1121 & 50.7 & 61.6 & & 60.5 & & 1341 & 49.3 & 60.4 & & 56.0 & \\
\hline 1 & 3647 & 37.1 & 62.9 & & 59.7 & & 1643 & 48.6 & 64.1 & & 63.3 & & 2004 & 51.4 & 61.8 & & 56.2 & \\
\hline$\geq 2$ & 4133 & 36.3 & 61.6 & & 54.8 & & 1693 & 42.8 & 63.6 & & 59.5 & & 2440 & 57.2 & 60.2 & & 51.3 & \\
\hline $\begin{array}{l}\text { Average age of offspring } \\
\text { (years) }\end{array}$ & & & & $<0.0001$ & & $<0.0001$ & & & & $<0.0001$ & & $<0.0001$ & & & & & & $<0.0001$ \\
\hline Q1 $(\leq 27.5)$ & 3487 & 45.0 & 64.2 & & 63.0 & & 1805 & 57.1 & 64.3 & & 64.2 & & 1682 & 42.9 & 64.1 & & 61.4 & \\
\hline Q2 (27.6-36.0) & 2637 & 25.3 & 63.1 & & 58.8 & & 1216 & 46.0 & 64.4 & & 61.8 & & 1421 & 54.0 & 62.0 & & 56.3 & \\
\hline Q3 (36.1-44.0) & 2246 & 17.2 & 58.4 & & 50.3 & & 904 & 37.8 & 59.7 & & 54.6 & & 1342 & 62.3 & 57.5 & & 47.8 & \\
\hline Q4 ( $\geq 44.1)$ & 1872 & 12.5 & 56.6 & & 44.9 & & 532 & 25.8 & 58.0 & & 49.1 & & 1340 & 74.2 & 56.2 & & 43.5 & \\
\hline Total & 10242 & 100.0 & 62.0 & & 57.5 & & 4457 & 47.1 & 63.2 & & 61.3 & & 5785 & 53.0 & 60.8 & & 54.2 & \\
\hline
\end{tabular}


Table 2 Adjusted effect of number of offspring on HRQoL according to parent

\begin{tabular}{|c|c|c|c|c|c|c|c|c|c|}
\hline & \multicolumn{3}{|l|}{ Total } & \multicolumn{3}{|l|}{ Father } & \multicolumn{3}{|l|}{ Mother } \\
\hline & Estimate & SE & p Value & Estimate & SE & p Value & Estimate & SE & p Value \\
\hline \multicolumn{10}{|l|}{ Number of offspring } \\
\hline 0 & -7.762 & 0.679 & $<0.0001$ & -6.143 & 0.949 & $<0.0001$ & -8.372 & 1.031 & $<0.0001$ \\
\hline 1 & -2.425 & 0.398 & $<0.0001$ & -2.245 & 0.589 & 0.000 & -2.554 & 0.543 & $<0.0001$ \\
\hline 2 & Ref. & & & Ref. & & & Ref. & & \\
\hline 3 & 0.991 & 0.279 & 0.000 & 1.111 & 0.412 & 0.008 & 0.967 & 0.382 & 0.012 \\
\hline 4 & -0.744 & 0.379 & 0.051 & -0.418 & 0.605 & 0.491 & -0.785 & 0.488 & 0.109 \\
\hline$\geq 5$ & -1.529 & 0.414 & 0.000 & -0.400 & 0.700 & 0.569 & -1.897 & 0.516 & 0.000 \\
\hline \multicolumn{10}{|l|}{ Average age of offspring (years) } \\
\hline Q1 $(\leq 27.5)$ & -1.333 & 0.677 & 0.049 & -2.467 & 1.097 & 0.025 & -1.283 & 0.907 & 0.158 \\
\hline Q2 (27.6-36.0) & -0.271 & 0.576 & 0.638 & -1.081 & 0.977 & 0.269 & -1.297 & 0.757 & 0.087 \\
\hline Q3 (36.1-44.0) & 0.123 & 0.465 & 0.793 & -0.123 & 0.821 & 0.881 & -0.527 & 0.593 & 0.374 \\
\hline Q4 $(\geq 44.1)$ & Ref. & & & Ref. & & & Ref. & & \\
\hline \multicolumn{10}{|l|}{ Age (years) } \\
\hline$\leq 49$ & Ref. & & & Ref. & & & Ref. & & \\
\hline $50-54$ & 0.172 & 0.426 & 0.687 & 0.006 & 0.621 & 0.993 & 0.745 & 0.597 & 0.212 \\
\hline $55-59$ & -0.537 & 0.500 & 0.283 & -0.385 & 0.705 & 0.585 & 0.118 & 0.741 & 0.874 \\
\hline $60-64$ & -1.612 & 0.559 & 0.004 & -1.129 & 0.815 & 0.166 & -1.425 & 0.815 & 0.081 \\
\hline $65-69$ & -2.771 & 0.629 & $<0.0001$ & -1.055 & 0.921 & 0.252 & -4.164 & 0.909 & $<0.0001$ \\
\hline $70-74$ & -4.546 & 0.711 & $<0.0001$ & -3.596 & 1.062 & 0.001 & -5.740 & 1.009 & $<0.0001$ \\
\hline$\geq 75$ & -6.028 & 0.782 & $<0.0001$ & -6.268 & 1.250 & $<0.0001$ & -7.011 & 1.069 & $<0.0001$ \\
\hline \multicolumn{10}{|l|}{ Gender } \\
\hline Male (father) & 0.479 & 0.326 & 0.142 & NA & & & NA & & \\
\hline Female (mother) & Ref. & & & & & & & & \\
\hline \multicolumn{10}{|l|}{ Education } \\
\hline Elementary school or less & -11.304 & 0.397 & $<0.0001$ & -9.976 & 0.517 & 0.000 & -13.314 & 0.689 & $<0.0001$ \\
\hline Middle school & -6.683 & 0.405 & $<0.0001$ & -6.399 & 0.528 & 0.001 & -8.271 & 0.705 & $<0.0001$ \\
\hline High school & -3.879 & 0.361 & $<0.0001$ & -4.015 & 0.445 & 0.003 & -5.078 & 0.670 & 0.000 \\
\hline College or above & Ref. & & & Ref. & & & Ref. & & \\
\hline \multicolumn{10}{|l|}{ Marital status } \\
\hline Married & 2.615 & 0.296 & $<0.0001$ & 4.332 & 0.595 & $<0.0001$ & 1.460 & 0.350 & $<0.0001$ \\
\hline Single (including separated, divorced) & Ref. & & & Ref. & & & Ref. & & \\
\hline \multicolumn{10}{|l|}{ Income } \\
\hline Yes & 1.871 & 0.278 & $<0.0001$ & 2.493 & 0.364 & $<0.0001$ & 1.178 & 0.442 & 0.008 \\
\hline No & Ref. & & & Ref. & & & Ref. & & \\
\hline \multicolumn{10}{|l|}{ Economic activity } \\
\hline Yes & 4.876 & 0.261 & $<0.0001$ & 7.007 & 0.400 & $<0.0001$ & 3.300 & 0.356 & $<0.0001$ \\
\hline No & Ref. & & & Ref. & & & Ref. & & \\
\hline \multicolumn{10}{|l|}{ Number of visits with friends } \\
\hline Never & -11.696 & 0.418 & $<0.0001$ & -12.802 & 0.637 & $<0.0001$ & -10.127 & 0.558 & $<0.0001$ \\
\hline 3-6 times a year & -5.672 & 0.420 & $<0.0001$ & -5.831 & 0.635 & $<0.0001$ & -5.447 & 0.560 & $<0.0001$ \\
\hline $1-2$ times a month & -3.538 & 0.303 & $<0.0001$ & -3.485 & 0.446 & $<0.0001$ & -3.694 & 0.420 & $<0.0001$ \\
\hline $1-2$ times a week & -2.609 & 0.263 & $<0.0001$ & -3.035 & 0.416 & $<0.0001$ & -2.319 & 0.337 & $<0.0001$ \\
\hline Every day & Ref. & & & Ref. & & & Ref. & & \\
\hline Smoking status & & & & & & & & & \\
\hline Never & 2.259 & 0.334 & $<0.0001$ & 1.664 & 0.385 & $<0.0001$ & 4.339 & 0.840 & $<0.0001$ \\
\hline Former smoker & 1.592 & 0.377 & $<0.0001$ & 1.551 & 0.407 & 0.000 & -0.292 & 1.471 & 0.843 \\
\hline Smoker & Ref. & & & Ref. & & & Ref. & & \\
\hline Alcohol use & & & & & & & & & \\
\hline Yes & 1.904 & 0.267 & $<0.0001$ & 1.674 & 0.413 & $<0.0001$ & 2.084 & 0.361 & $<0.0001$ \\
\hline Former user & -3.505 & 0.382 & $<0.0001$ & -3.529 & 0.534 & $<0.0001$ & -3.380 & 0.619 & $<0.0001$ \\
\hline No & Ref. & & & Ref. & & & Ref. & & \\
\hline Depressive symptoms & & & & & & & & & \\
\hline Yes & -13.504 & 0.404 & $<0.0001$ & -13.378 & 0.708 & $<0.0001$ & -13.206 & 0.487 & $<0.0001$ \\
\hline No & Ref. & & & Ref. & & & Ref. & & \\
\hline Number of chronic diseases & & & & & & & & & \\
\hline 0 & 12.557 & 0.518 & $<0.0001$ & 12.626 & 0.836 & $<0.0001$ & 12.118 & 0.657 & $<0.0001$ \\
\hline 1 & 7.116 & 0.548 & $<0.0001$ & 7.569 & 0.890 & $<0.0001$ & 6.591 & 0.690 & $<0.0001$ \\
\hline$\geq 2$ & Ref. & & & Ref. & & & Ref. & & \\
\hline Year & & & & & & & & & \\
\hline 2006 & -3.963 & 0.335 & $<0.0001$ & -4.284 & 0.499 & $<0.0001$ & -3.473 & 0.452 & $<0.0001$ \\
\hline 2008 & -4.275 & 0.327 & $<0.0001$ & -4.540 & 0.490 & $<0.0001$ & -3.829 & 0.440 & $<0.0001$ \\
\hline 2010 & -4.523 & 0.315 & $<0.0001$ & -4.733 & 0.471 & $<0.0001$ & -4.119 & 0.425 & $<0.0001$ \\
\hline 2012 & Ref. & & & Ref. & & & Ref. & & \\
\hline
\end{tabular}

HRQoL, health-related quality of life; Ref., reference. 
Table 3 Adjusted effect of number of offspring on QoL according to parent

\begin{tabular}{|c|c|c|c|c|c|c|c|c|c|}
\hline & \multicolumn{3}{|l|}{ Total } & \multicolumn{3}{|l|}{ Father } & \multicolumn{3}{|l|}{ Mother } \\
\hline & Estimate & SE & p Value & Estimate & SE & p Value & Estimate & SE & p Value \\
\hline \multicolumn{10}{|l|}{ Number of offspring } \\
\hline 0 & -9.384 & 0.606 & $<0.0001$ & -7.492 & 0.829 & $<0.0001$ & -8.998 & 0.934 & $<0.0001$ \\
\hline 1 & -2.570 & 0.355 & $<0.0001$ & -2.515 & 0.515 & $<0.0001$ & -2.422 & 0.492 & $<0.0001$ \\
\hline 2 & Ref. & & & Ref. & & & Ref. & & \\
\hline 3 & 1.162 & 0.249 & $<0.0001$ & 1.470 & 0.360 & $<0.0001$ & 1.020 & 0.346 & 0.004 \\
\hline 4 & -0.339 & 0.338 & 0.316 & 0.404 & 0.528 & 0.446 & -0.611 & 0.442 & 0.168 \\
\hline$\geq 5$ & 0.885 & 0.369 & 0.017 & 1.304 & 0.612 & 0.035 & 0.797 & 0.467 & 0.090 \\
\hline \multicolumn{10}{|l|}{ Average age of offspring (years) } \\
\hline Q1 $(\leq 27.5)$ & -4.221 & 0.604 & $<0.0001$ & -4.847 & 0.958 & $<0.0001$ & -4.473 & 0.822 & $<0.0001$ \\
\hline Q2 (27.6-36.0) & -2.427 & 0.514 & $<0.0001$ & -2.827 & 0.854 & 0.001 & -2.949 & 0.686 & $<0.0001$ \\
\hline Q3 (36.1-44.0) & -1.895 & 0.415 & $<0.0001$ & -2.012 & 0.717 & 0.005 & -1.814 & 0.538 & 0.001 \\
\hline Q4 ( $\geq 44.1)$ & Ref. & & & Ref. & & & Ref. & & \\
\hline \multicolumn{10}{|l|}{ Age (years) } \\
\hline$\leq 49$ & Ref. & & & Ref. & & & Ref. & & \\
\hline $50-54$ & -0.383 & 0.380 & 0.314 & -0.670 & 0.543 & 0.217 & -0.077 & 0.541 & 0.887 \\
\hline $55-59$ & -0.006 & 0.446 & 0.989 & -0.151 & 0.616 & 0.806 & -0.005 & 0.671 & 0.994 \\
\hline $60-64$ & -0.529 & 0.499 & 0.289 & -0.539 & 0.712 & 0.449 & -0.933 & 0.739 & 0.206 \\
\hline $65-69$ & -1.508 & 0.561 & 0.007 & -0.910 & 0.805 & 0.258 & -2.876 & 0.823 & 0.001 \\
\hline $70-74$ & -3.509 & 0.634 & $<0.0001$ & -3.794 & 0.928 & $<0.0001$ & -4.213 & 0.914 & $<0.0001$ \\
\hline$\geq 75$ & -4.518 & 0.698 & $<0.0001$ & -5.692 & 1.092 & $<0.0001$ & -5.202 & 0.968 & $<0.0001$ \\
\hline \multicolumn{10}{|l|}{ Gender } \\
\hline Male (father) & -0.044 & 0.291 & 0.880 & NA & & & NA & & \\
\hline Female (mother) & Ref. & & & & & & & & \\
\hline \multicolumn{10}{|l|}{ Education } \\
\hline Elementary school or less & -10.518 & 0.354 & $<0.0001$ & -9.596 & 0.452 & 0.000 & -11.638 & 0.624 & $<0.0001$ \\
\hline Middle school & -6.714 & 0.362 & $<0.0001$ & -6.580 & 0.462 & 0.001 & -7.228 & 0.639 & $<0.0001$ \\
\hline High school & -3.891 & 0.322 & $<0.0001$ & -3.959 & 0.389 & 0.002 & -4.407 & 0.607 & 0.000 \\
\hline College or above & Ref. & & & Ref. & & & Ref. & & \\
\hline \multicolumn{10}{|l|}{ Marital status } \\
\hline Married & 0.089 & 0.248 & 0.718 & 0.983 & 0.318 & 0.002 & -1.249 & 0.400 & 0.002 \\
\hline Single (including separated, divorced) & Ref. & & & Ref. & & & Ref. & & \\
\hline \multicolumn{10}{|l|}{ Income } \\
\hline Yes & 5.149 & 0.264 & $<0.0001$ & 9.107 & 0.520 & $<0.0001$ & 3.625 & 0.317 & $<0.0001$ \\
\hline No & Ref. & & & Ref. & & & Ref. & & \\
\hline \multicolumn{10}{|l|}{ Economic activity } \\
\hline Yes & 2.949 & 0.233 & $<0.0001$ & 4.687 & 0.350 & $<0.0001$ & 1.503 & 0.323 & $<0.0001$ \\
\hline No & Ref. & & & Ref. & & & Ref. & & \\
\hline \multicolumn{10}{|l|}{ Number of visits with friends } \\
\hline Never & -11.104 & 0.373 & $<0.0001$ & -11.997 & 0.556 & $<0.0001$ & -9.578 & 0.505 & $<0.0001$ \\
\hline 3-6 times a year & -5.126 & 0.375 & $<0.0001$ & -5.246 & 0.555 & $<0.0001$ & -4.901 & 0.507 & $<0.0001$ \\
\hline $1-2$ times a month & -3.924 & 0.271 & $<0.0001$ & -3.770 & 0.390 & $<0.0001$ & -4.120 & 0.380 & $<0.0001$ \\
\hline $1-2$ times a week & -2.644 & 0.234 & $<0.0001$ & -2.547 & 0.363 & $<0.0001$ & -2.757 & 0.305 & $<0.0001$ \\
\hline Every day & Ref. & & & Ref. & & & Ref. & & \\
\hline Smoking status & & & & & & & & & \\
\hline Never & 3.988 & 0.298 & $<0.0001$ & 3.349 & 0.336 & $<0.0001$ & 6.662 & 0.761 & $<0.0001$ \\
\hline Former smoker & 3.142 & 0.336 & $<0.0001$ & 2.972 & 0.356 & $<0.0001$ & 3.185 & 1.333 & 0.018 \\
\hline Smoker & Ref. & & & Ref. & & & Ref. & & \\
\hline Alcohol use & & & & & & & & & \\
\hline Yes & 0.746 & 0.238 & 0.002 & 0.070 & 0.360 & 0.845 & 1.332 & 0.327 & $<0.0001$ \\
\hline Former user & -2.202 & 0.341 & $<0.0001$ & -2.296 & 0.466 & $<0.0001$ & -2.038 & 0.561 & 0.000 \\
\hline No & Ref. & & & Ref. & & & Ref. & & \\
\hline Depressive symptoms & & & & & & & & & \\
\hline Yes & -11.078 & 0.360 & $<0.0001$ & -10.222 & 0.618 & $<0.0001$ & -11.179 & 0.441 & $<0.0001$ \\
\hline No & Ref. & & & Ref. & & & Ref. & & \\
\hline Number of chronic diseases & & & & & & & & & \\
\hline 0 & 4.211 & 0.462 & $<0.0001$ & 3.918 & 0.730 & $<0.0001$ & 4.379 & 0.595 & $<0.0001$ \\
\hline 1 & 2.581 & 0.489 & $<0.0001$ & 2.803 & 0.778 & 0.000 & 2.457 & 0.625 & $<0.0001$ \\
\hline$\geq 2$ & Ref. & & & Ref. & & & Ref. & & \\
\hline Year & & & & & & & & & \\
\hline 2006 & -3.798 & 0.299 & $<0.0001$ & -3.127 & 0.436 & $<0.0001$ & -4.103 & 0.410 & $<0.0001$ \\
\hline 2008 & -2.769 & 0.292 & $<0.0001$ & -2.320 & 0.428 & $<0.0001$ & -2.891 & 0.398 & $<0.0001$ \\
\hline 2010 & -3.100 & 0.281 & $<0.0001$ & -2.806 & 0.411 & $<0.0001$ & -3.135 & 0.385 & $<0.0001$ \\
\hline 2012 & Ref. & & & Ref. & & & Ref. & & \\
\hline
\end{tabular}

QoL, quality of life; Ref., reference. 


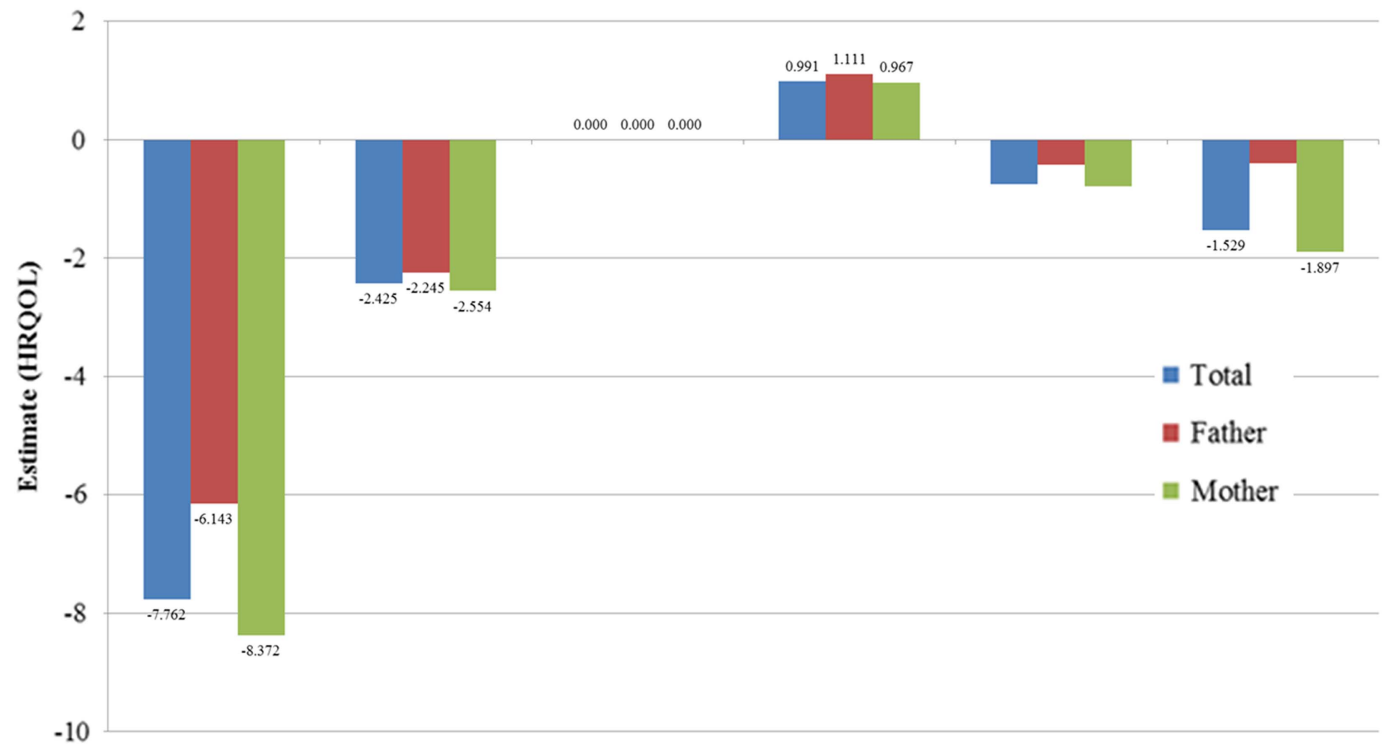

Number of offspring

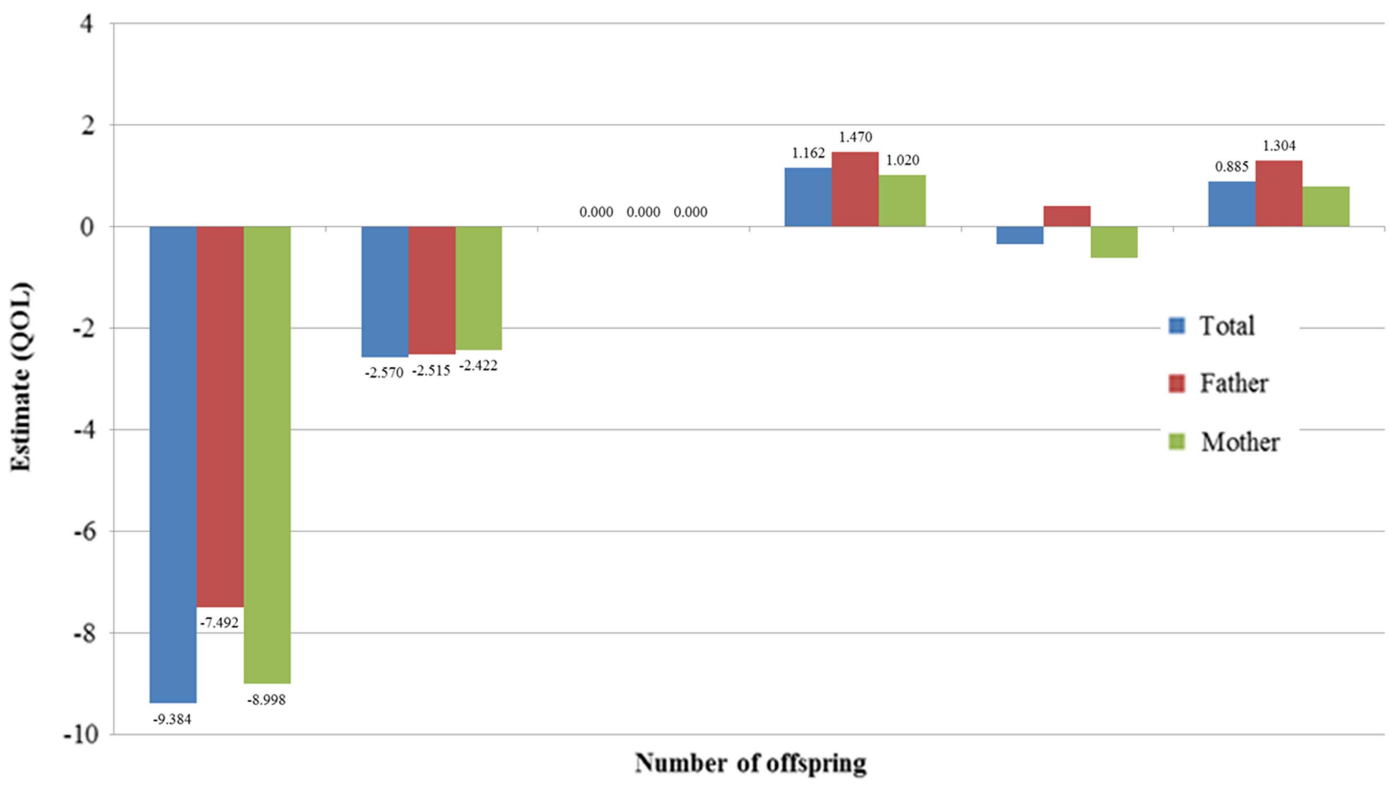

Figure 1 Adjusted effects of number of offspring on health-related quality of life (HRQoL) and QoL, by parent. Adjusted for average age of offspring, age, gender, education, marital status, income, economic activity, number of visits with friends, smoking status, alcohol use, depressive symptoms, number of chronic diseases and year.

For mothers with no boys and no girls, the estimates for HRQoL and QoL were -8.392 and -9.108 , respectively (SE: 1.054 and 0.955 ; both $\mathrm{p}<0.0001$ ) compared with fathers with one boy and one girl. For fathers with two or more boys and two or more girls, the estimates for HRQoL and QoL were -1.249 and -0.152 , respectively (SE: 0.541 and $0.490 ; \mathrm{p}=0.024$ and $\mathrm{p}=0.502$ ) compared with mothers with one boy and one girl.

\section{DISCUSSION}

Our primary purpose in this study was to investigate the effect of number of offspring on HRQoL and QoL in a longitudinal model. We also sought to investigate any association between composition of offspring and
HRQoL and QoL. We used a nationally representative sample of older adults ( $\geq 45$ years of age) in South Korea.

Overall, for number of offspring, the HRQoL and QoL estimates varied more for mothers than for fathers (tables 2 and 3). There was a similar tendency for composition of offspring (tables 4 and 5). Although having more offspring tended to reduce HRQoL compared with QoL, both HRQoL and QoL were decreased for those with no offspring. Additionally, the decrease in HRQoL was less than the decrease in QoL for fathers and mothers. Although the trends were similar for mothers and fathers, the deterioration in HRQoL and QoL was greater for mothers than for fathers. Tables 4 and 5 show that the effects of the composition of 

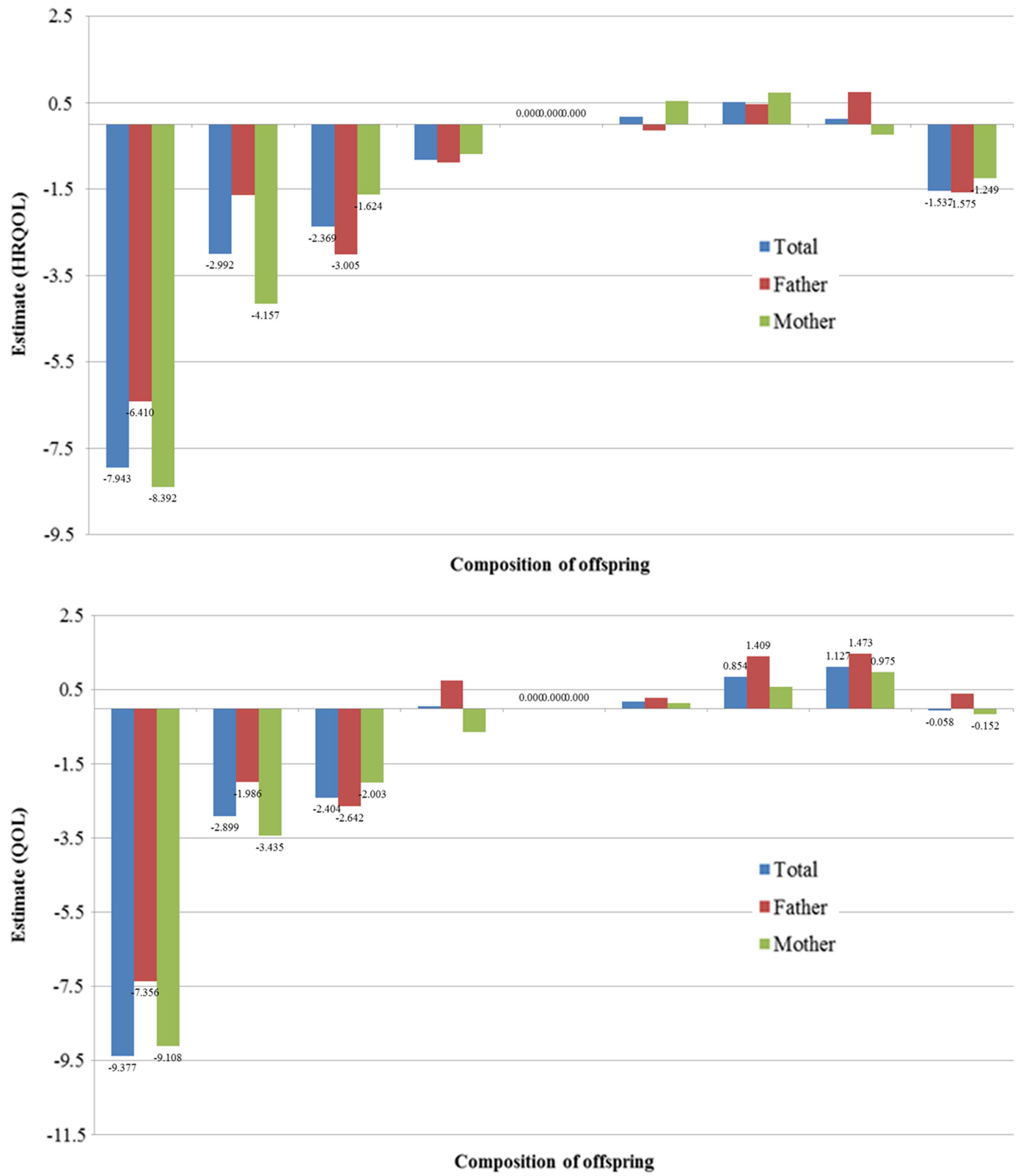

Figure 2 Adjusted effects of composition of offspring on health-related quality of life (HRQLL) and QoL, by parent. Adjusted for average age of offspring, age, gender, education, marital status, income, economic activity, number of visits with friends, smoking status, alcohol use, depressive symptoms, number of chronic diseases and year.

offspring on parents' HRQoL and QoL were inconsistent, but having more offspring tended to result in a greater deterioration in HRQoL (estimate: -1.537; $\mathrm{p}<0.001)$ compared with QoL (estimate: -0.058 ; $\mathrm{p}=0.878)$.

These associations were independent of offspringrelated variables (average age of offspring), sociodemographic variables (age, gender, education, marital status, numbers of visits with friends, income, and economic activity status), health risk behaviour variables (smoking status and alcohol consumption), health status (depression symptoms and number of chronic diseases) and year.

Well-being, such as eudaemonic well-being, is a concept that goes beyond subjective happiness and is not simply defined in terms of living well. Rather, the concept implies objective indicators of one's wellness and suggests human flourishing. Rogers ${ }^{19}$ argued that the eudaemonic position of well-being is the extent to which a person is fully functioning, rather than simply feeling pleasant. The eudaemonic paradigm defines well-being in terms of values and purposes in life and/ or potential achieving, with respect to a higher order of the self (ie, realisation of true self, positive relationships, human strengths and virtues), which reflect psychological well-being. ${ }^{20}$ Such themes have gained increasing appreciation in the aging-related literature. ${ }^{20}$

A recent study ${ }^{21}$ indicated that $\sim 20 \%$ of Korean older adults experience depression that interferes with 
Table 4 Adjusted effect of composition of offspring on HRQoL

\begin{tabular}{|c|c|c|c|c|c|c|c|c|c|}
\hline & \multicolumn{3}{|l|}{ Total } & \multicolumn{3}{|l|}{ Father } & \multicolumn{3}{|l|}{ Mother } \\
\hline & Estimate & SE & p Value & Estimate & SE & p Value & Estimate & SE & p Value \\
\hline \multicolumn{10}{|l|}{ Composition of offspring } \\
\hline 0 Boy and 0 girl & -7.943 & 0.697 & $<0.0001$ & -6.410 & 0.975 & $<0.0001$ & -8.392 & 1.054 & $<0.0001$ \\
\hline 0 Boy and 1 girl & -2.992 & 0.650 & $<0.0001$ & -1.640 & 0.974 & 0.095 & -4.157 & 0.873 & $<0.0001$ \\
\hline 1 Boy and 0 girl & -2.369 & 0.504 & $<0.0001$ & -3.005 & 0.740 & $<0.0001$ & -1.624 & 0.691 & 0.020 \\
\hline 0 Boy and $\geq 2$ girls & -0.824 & 0.435 & 0.059 & -0.884 & 0.624 & 0.159 & -0.685 & 0.609 & 0.263 \\
\hline 1 Boy and 1 girl & Ref. & & & Ref. & & & Ref. & & \\
\hline$\geq 2$ Boys and 0 girl & 0.173 & 0.337 & 0.607 & -0.142 & 0.484 & 0.770 & 0.539 & 0.471 & 0.253 \\
\hline$\geq 2$ Boys and 1 girl & 0.512 & 0.389 & 0.189 & 0.470 & 0.594 & 0.430 & 0.734 & 0.519 & 0.159 \\
\hline 1 Boy and $\geq 2$ girls & 0.131 & 0.354 & 0.712 & 0.744 & 0.524 & 0.158 & -0.238 & 0.481 & 0.622 \\
\hline$\geq 2$ Boys and $\geq 2$ girls & -1.537 & 0.422 & 0.000 & -1.575 & 0.689 & 0.024 & -1.249 & 0.541 & 0.022 \\
\hline \multicolumn{10}{|l|}{ Average age of offspring (years) } \\
\hline Q1 $(\leq 27.5)$ & -1.172 & 0.680 & 0.085 & -2.567 & 1.100 & 0.020 & -1.083 & 0.911 & 0.235 \\
\hline Q2 (27.6-36.0) & -0.111 & 0.577 & 0.848 & -1.154 & 0.977 & 0.238 & -1.099 & 0.759 & 0.148 \\
\hline Q3 (36.1-44.0) & 0.202 & 0.466 & 0.664 & -0.165 & 0.820 & 0.840 & -0.459 & 0.594 & 0.440 \\
\hline Q4 ( $\geq 44.1)$ & Ref. & & & Ref. & & & Ref. & & \\
\hline \multicolumn{10}{|l|}{ Age (years) } \\
\hline$\leq 49$ & Ref. & & & Ref. & & & Ref. & & \\
\hline $50-54$ & 0.158 & 0.426 & 0.711 & -0.035 & 0.621 & 0.955 & 0.727 & 0.597 & 0.224 \\
\hline $55-59$ & -0.527 & 0.501 & 0.293 & -0.449 & 0.707 & 0.525 & 0.129 & 0.742 & 0.862 \\
\hline $60-64$ & -1.566 & 0.560 & 0.005 & -1.147 & 0.817 & 0.160 & -1.384 & 0.815 & 0.090 \\
\hline $65-69$ & -2.757 & 0.629 & $<0.0001$ & -1.059 & 0.923 & 0.251 & -4.243 & 0.908 & $<0.0001$ \\
\hline $70-74$ & -4.632 & 0.709 & $<0.0001$ & -3.613 & 1.061 & 0.001 & -5.994 & 1.005 & $<0.0001$ \\
\hline$\geq 75$ & -6.194 & 0.779 & $<0.0001$ & -6.236 & 1.245 & $<0.0001$ & -7.396 & 1.063 & $<0.0001$ \\
\hline \multicolumn{10}{|l|}{ Gender } \\
\hline Male (father) & 0.513 & 0.326 & 0.116 & NA & & & NA & & \\
\hline Female (mother) & Ref. & & & & & & & & \\
\hline \multicolumn{10}{|l|}{ Education } \\
\hline Elementary school or less & -11.356 & 0.396 & $<0.0001$ & -9.956 & 0.517 & 0.000 & -13.431 & 0.689 & $<0.0001$ \\
\hline Middle school & -6.735 & 0.406 & $<0.0001$ & -6.431 & 0.529 & 0.001 & -8.370 & 0.706 & $<0.0001$ \\
\hline High school & -3.885 & 0.361 & $<0.0001$ & -4.002 & 0.445 & 0.003 & -5.136 & 0.670 & 0.000 \\
\hline College or above & Ref. & & & Ref. & & & Ref. & & \\
\hline \multicolumn{10}{|l|}{ Marital status } \\
\hline Married & 2.647 & 0.296 & $<0.0001$ & 4.384 & 0.595 & $<0.0001$ & 1.476 & 0.350 & $<0.0001$ \\
\hline Single (including separated, divorced) & Ref. & & & Ref. & & & Ref. & & \\
\hline \multicolumn{10}{|l|}{ Previous year income } \\
\hline Yes & 1.896 & 0.278 & $<0.0001$ & 2.493 & 0.364 & $<0.0001$ & 1.208 & 0.442 & 0.006 \\
\hline No & Ref. & & & Ref. & & & Ref. & & \\
\hline Economic activity & & & & & & & & & \\
\hline Yes & 4.820 & 0.261 & $<0.0001$ & 6.993 & 0.400 & $<0.0001$ & 3.233 & 0.356 & $<0.0001$ \\
\hline No & Ref. & & & Ref. & & & Ref. & & \\
\hline Number of visits with friends & & & & & & & & & \\
\hline Never & -11.684 & 0.418 & $<0.0001$ & -12.780 & 0.637 & $<0.0001$ & -10.093 & 0.558 & $<0.0001$ \\
\hline $3-6$ times a year & -5.641 & 0.420 & $<0.0001$ & -5.845 & 0.635 & $<0.0001$ & -5.406 & 0.560 & $<0.0001$ \\
\hline $1-2$ times a month & -3.553 & 0.304 & $<0.0001$ & -3.516 & 0.446 & $<0.0001$ & -3.698 & 0.420 & $<0.0001$ \\
\hline 1-2 times a week & -2.616 & 0.263 & $<0.0001$ & -3.068 & 0.416 & $<0.0001$ & -2.307 & 0.337 & $<0.0001$ \\
\hline Every day & Ref. & & & Ref. & & & Ref. & & \\
\hline Smoking status & & & & & & & & & \\
\hline Never & 2.282 & 0.334 & $<0.0001$ & 1.655 & 0.385 & $<0.0001$ & 4.332 & 0.841 & $<0.0001$ \\
\hline Former smoker & 1.612 & 0.377 & $<0.0001$ & 1.523 & 0.408 & 0.000 & -0.225 & 1.472 & 0.879 \\
\hline Smoker & Ref. & & & Ref. & & & Ref. & & \\
\hline Alcohol use & & & & & & & & & \\
\hline Yes & 1.885 & 0.267 & $<0.0001$ & 1.636 & 0.413 & $<0.0001$ & 2.040 & 0.361 & $<0.0001$ \\
\hline Former user & -3.510 & 0.383 & $<0.0001$ & -3.576 & 0.534 & $<0.0001$ & -3.367 & 0.620 & $<0.0001$ \\
\hline No & Ref. & & & Ref. & & & Ref. & & \\
\hline Depressive symptoms & & & & & & & & & \\
\hline Yes & -13.491 & 0.404 & $<0.0001$ & -13.388 & 0.708 & $<0.0001$ & -13.181 & 0.487 & $<0.0001$ \\
\hline No & Ref. & & & Ref. & & & Ref. & & \\
\hline Number of chronic diseases & & & & & & & & & \\
\hline 0 & 12.578 & 0.518 & $<0.0001$ & 12.573 & 0.836 & $<0.0001$ & 12.171 & 0.657 & $<0.0001$ \\
\hline 1 & 7.151 & 0.548 & $<0.0001$ & 7.535 & 0.891 & $<0.0001$ & 6.649 & 0.690 & $<0.0001$ \\
\hline$\geq 2$ & Ref. & & & Ref. & & & Ref. & & \\
\hline Year & & & & & & & & & \\
\hline 2006 & -3.912 & 0.334 & $<0.0001$ & -4.280 & 0.499 & $<0.0001$ & -3.381 & 0.452 & $<0.0001$ \\
\hline 2008 & -4.243 & 0.327 & $<0.0001$ & -4.530 & 0.490 & $<0.0001$ & -3.769 & 0.440 & $<0.0001$ \\
\hline 2010 & -4.506 & 0.316 & $<0.0001$ & -4.718 & 0.471 & $<0.0001$ & -4.088 & 0.425 & $<0.0001$ \\
\hline 2012 & Ref. & & & Ref. & & & Ref. & & \\
\hline
\end{tabular}

HRQoL, health-related quality of life; Ref., reference. 
Table 5 Adjusted effect of number of offspring on QoL according to parent

\begin{tabular}{|c|c|c|c|c|c|c|c|c|c|}
\hline & \multicolumn{3}{|l|}{ Total } & \multicolumn{3}{|l|}{ Father } & \multicolumn{3}{|l|}{ Mother } \\
\hline & Estimate & SE & p Value & Estimate & SE & p Value & Estimate & SE & p Value \\
\hline \multicolumn{10}{|l|}{ Composition of offspring } \\
\hline 0 Boy and 0 girl & -9.377 & 0.621 & $<0.0001$ & -7.356 & 0.852 & $<0.0001$ & -9.108 & 0.955 & $<0.0001$ \\
\hline 0 Boy and 1 girl & -2.899 & 0.579 & $<0.0001$ & -1.986 & 0.851 & 0.021 & -3.435 & 0.790 & $<0.0001$ \\
\hline 1 Boy and 0 girl & -2.404 & 0.450 & $<0.0001$ & -2.642 & 0.647 & $<0.0001$ & -2.003 & 0.626 & 0.002 \\
\hline 0 Boy and $\geq 2$ girls & 0.059 & 0.388 & 0.880 & 0.749 & 0.545 & 0.172 & -0.639 & 0.552 & 0.248 \\
\hline 1 Boy and 1 girl & Ref. & & & Ref. & & & Ref. & & \\
\hline$\geq 2$ Boys and 0 girl & 0.188 & 0.301 & 0.532 & 0.287 & 0.423 & 0.499 & 0.151 & 0.426 & 0.723 \\
\hline$\geq 2$ Boys and 1 girl & 0.854 & 0.347 & 0.015 & 1.409 & 0.519 & 0.008 & 0.580 & 0.470 & 0.218 \\
\hline 1 Boy and $\geq 2$ girls & 1.127 & 0.315 & 0.000 & 1.473 & 0.458 & 0.002 & 0.975 & 0.436 & 0.027 \\
\hline$\geq 2$ Boys and $\geq 2$ girls & -0.058 & 0.377 & 0.878 & 0.405 & 0.602 & 0.502 & -0.152 & 0.490 & 0.757 \\
\hline \multicolumn{10}{|l|}{ Average age of offspring (years) } \\
\hline Q1 $(\leq 27.5)$ & -4.292 & 0.606 & $<0.0001$ & -5.075 & 0.961 & $<0.0001$ & -4.468 & 0.825 & $<0.0001$ \\
\hline Q2 (27.6-36.0) & -2.500 & 0.515 & $<0.0001$ & -3.009 & 0.854 & 0.000 & -2.963 & 0.688 & $<0.0001$ \\
\hline Q3 (36.1-44.0) & -1.950 & 0.415 & $<0.0001$ & -2.104 & 0.717 & 0.003 & -1.857 & 0.538 & 0.001 \\
\hline Q4 ( $\geq 44.1)$ & Ref. & & & Ref. & & & Ref. & & \\
\hline \multicolumn{10}{|l|}{ Age (years) } \\
\hline$\leq 49$ & Ref. & & & Ref. & & & Ref. & & \\
\hline $50-54$ & -0.386 & 0.380 & 0.310 & -0.685 & 0.543 & 0.207 & -0.067 & 0.541 & 0.902 \\
\hline $55-59$ & -0.003 & 0.447 & 0.995 & -0.177 & 0.617 & 0.774 & 0.002 & 0.672 & 0.997 \\
\hline $60-64$ & -0.515 & 0.499 & 0.303 & -0.543 & 0.714 & 0.447 & -0.925 & 0.739 & 0.211 \\
\hline $65-69$ & -1.476 & 0.561 & 0.009 & -0.916 & 0.807 & 0.256 & -2.835 & 0.822 & 0.001 \\
\hline $70-74$ & -3.468 & 0.633 & $<0.0001$ & -3.779 & 0.927 & $<0.0001$ & -4.160 & 0.910 & $<0.0001$ \\
\hline$\geq 75$ & -4.386 & 0.695 & $<0.0001$ & -5.601 & 1.088 & $<0.0001$ & -5.053 & 0.962 & $<0.0001$ \\
\hline \multicolumn{10}{|l|}{ Gender } \\
\hline Male (father) & -0.045 & 0.291 & 0.877 & NA & & & NA & & \\
\hline Female (mother) & Ref. & & & & & & & & \\
\hline \multicolumn{10}{|l|}{ Education } \\
\hline Elementary school or less & -10.516 & 0.354 & $<0.0001$ & -9.563 & 0.452 & 0.000 & -11.701 & 0.624 & $<0.0001$ \\
\hline Middle school & -6.758 & 0.362 & $<0.0001$ & -6.589 & 0.462 & 0.001 & -7.343 & 0.640 & $<0.0001$ \\
\hline High school & -3.910 & 0.322 & $<0.0001$ & -3.974 & 0.389 & 0.002 & -4.455 & 0.607 & 0.000 \\
\hline College or above & Ref. & & & Ref. & & & Ref. & & \\
\hline \multicolumn{10}{|l|}{ Marital status } \\
\hline Married & 5.170 & 0.264 & $<0.0001$ & 9.135 & 0.520 & $<0.0001$ & 3.638 & 0.317 & $<0.0001$ \\
\hline Single (including separated, divorced) & Ref. & & & Ref. & & & Ref. & & \\
\hline \multicolumn{10}{|l|}{ Previous year income } \\
\hline Yes & 0.092 & 0.248 & 0.711 & 0.985 & 0.318 & 0.002 & -1.270 & 0.400 & 0.002 \\
\hline No & Ref. & & & Ref. & & & Ref. & & \\
\hline \multicolumn{10}{|l|}{ Economic activity } \\
\hline Yes & 2.954 & 0.233 & $<0.0001$ & 4.710 & 0.350 & $<0.0001$ & 1.511 & 0.323 & $<0.0001$ \\
\hline No & Ref. & & & Ref. & & & Ref. & & \\
\hline \multicolumn{10}{|l|}{ Number of visits with friends } \\
\hline Never & -11.091 & 0.373 & $<0.0001$ & -11.978 & 0.557 & $<0.0001$ & -9.569 & 0.506 & $<0.0001$ \\
\hline 3-6 times a year & -5.100 & 0.375 & $<0.0001$ & -5.237 & 0.555 & $<0.0001$ & -4.884 & 0.507 & $<0.0001$ \\
\hline $1-2$ times a month & -3.937 & 0.271 & $<0.0001$ & -3.786 & 0.390 & $<0.0001$ & -4.142 & 0.380 & $<0.0001$ \\
\hline $1-2$ times a week & -2.655 & 0.234 & $<0.0001$ & -2.574 & 0.363 & $<0.0001$ & -2.760 & 0.305 & $<0.0001$ \\
\hline Every day & Ref. & & & Ref. & & & Ref. & & \\
\hline \multicolumn{10}{|l|}{ Smoking status } \\
\hline Never & 3.990 & 0.298 & $<0.0001$ & 3.345 & 0.336 & $<0.0001$ & 6.707 & 0.762 & $<0.0001$ \\
\hline Former smoker & 3.140 & 0.336 & $<0.0001$ & 2.971 & 0.357 & $<0.0001$ & 3.309 & 1.333 & 0.014 \\
\hline Smoker & Ref. & & & Ref. & & & Ref. & & \\
\hline \multicolumn{10}{|l|}{ Alcohol use } \\
\hline Yes & 0.738 & 0.239 & 0.002 & 0.033 & 0.361 & 0.926 & 1.355 & 0.327 & $<0.0001$ \\
\hline Former user & -2.209 & 0.341 & $<0.0001$ & -2.319 & 0.467 & $<0.0001$ & -2.012 & 0.561 & 0.000 \\
\hline No & Ref. & & & Ref. & & & Ref. & & \\
\hline \multicolumn{10}{|l|}{ Depressive symptoms } \\
\hline Yes & -11.104 & 0.360 & $<0.0001$ & -10.257 & 0.618 & $<0.0001$ & -11.184 & 0.441 & $<0.0001$ \\
\hline No & Ref. & & & Ref. & & & Ref. & & \\
\hline
\end{tabular}


Table 5 Continued

\begin{tabular}{|c|c|c|c|c|c|c|c|c|c|}
\hline & \multicolumn{3}{|l|}{ Total } & \multicolumn{3}{|l|}{ Father } & \multicolumn{3}{|l|}{ Mother } \\
\hline & Estimate & SE & p Value & Estimate & SE & p Value & Estimate & SE & p Value \\
\hline \multicolumn{10}{|c|}{ Number of chronic diseases } \\
\hline 0 & 4.205 & 0.462 & $<0.0001$ & 3.903 & 0.730 & $<0.0001$ & 4.376 & 0.595 & $<0.0001$ \\
\hline 1 & 2.582 & 0.489 & $<0.0001$ & 2.780 & 0.778 & 0.000 & 2.474 & 0.625 & $<0.0001$ \\
\hline$\geq 2$ & Ref. & & & Ref. & & & Ref. & & \\
\hline \multicolumn{10}{|l|}{ Year } \\
\hline 2006 & -3.821 & 0.298 & $<0.0001$ & -3.166 & 0.436 & $<0.0001$ & -4.117 & 0.409 & $<0.0001$ \\
\hline 2008 & -2.785 & 0.292 & $<0.0001$ & -2.347 & 0.428 & $<0.0001$ & -2.897 & 0.398 & $<0.0001$ \\
\hline 2010 & -3.105 & 0.282 & $<0.0001$ & -2.815 & 0.412 & $<0.0001$ & -3.131 & 0.385 & $<0.0001$ \\
\hline 2012 & Ref. & & & Ref. & & & Ref. & & \\
\hline
\end{tabular}

QoL, quality of life; Ref., reference.

their daily lives and continues for $>2$ weeks. Depression in old age threatens mental and physical health, and leads to a deterioration in QoL. ${ }^{22}$ Older adults in Korea who live alone are at risk of negative effects on their mental health; $15 \%$ use alcohol and $60 \%$ of these have an alcohol use problem. Moreover, their level of suicidal ideation is higher than that of the general population. $^{23}$

Living with family and having children could increase the QoL of older adults by providing social and psychological support. Using a meta-analysis approach, ${ }^{5}$ Pinquart and Sorensen examined 286 studies and found that there were positive relationships between the social status of elderly people, social relationships, competence and well-being. Chappell ${ }^{24}$ reported that social support and health contribute significantly to the variance in subjective QoL characteristics. Family cohesion and adaptability are also positively correlated with quality of life, and adaptability is also correlated with social support from the family. Such results are consistent with previous findings on the association between cohesion or adaptability and adjustment to serious illness. ${ }^{25} 26$

The term 'QoL' is used to describe the general wellbeing of populations and is employed in the international development, health and political domains. It should not be confused with the concept of 'standard of living', which is based primarily on income. Standard indicators of QoL include wealth and employment, and also the built environment, physical and mental health, education, recreation and leisure time, and social belonging variables. ${ }^{3}$ Health status is an important factor that directly affects QoL. However, HRQoL includes the individual's perception of his/her health status, and activity in physical, social and psychological terms.

Our previous studies ${ }^{17}$ revealed that there is a significant relationship between offspring and QoL characteristics. The present study indicates that offspring have statistically significant effects on HRQoL and QoL. For both of these indicators, there were differences between disease states and average total life quality scores.

This study has several strengths and limitations. A strength was that the survey participants were representative of the overall population. A large population sample size was used to estimate the effects, so the results can be generalised to the population of older adults aged $\geq 45$ years in South Korea. Nevertheless, possible sample biases could have been present in the study. First, respondents' reports were subjective and imperfect measures, potentially affected by perception bias and adaptation of resources. Second, because personality characteristics are likely to be associated with HRQoL and QoL characteristics, failure to include them in the statistical models may have resulted in exaggeration of the associations of interest. Third, in addition to the potential biases discussed above that were likely to inflate the associations between offspring and at least some of the health variables, we recognise that the estimates may have understated the potential associations for all of the outcomes because we used a short follow-up period in our analysis. Fourth, twins or triplets might have been present in the population, but we did not examine the composition of offspring because of insufficient sample sizes. Fifth, we used longitudinal data for our estimates, but the results possibly reflected reverse causality and bidirectional relationships when the associations between number of offspring and quality of life were assessed. Sixth, some recent investigations have focused on the QoL characteristics of infertile patients. ${ }^{27} 28$ A study of the psychosocial burden of infertility treatment revealed that there is a decline in mental health and social support and an increase in physical and social stress in those who continue to experience involuntary childlessness after 1 year of treatment. ${ }^{29}$ However, we could not determine whether adults with no offspring were childless as a result of infertility.

\section{Conclusions}

We provided additional evidence that supports the effects of offspring-related variables on HRQoL and QoL. Having a larger number of offspring appeared to reduce HRQoL more than QoL. However, parents with no offspring seemed to experience greater deterioration in QoL than HRQoL. Additionally, QoL in older people was affected by several variables (eg, age, educational level, marital status, income, economic activity, smoking status, alcohol use, depression symptoms, chronic 
disease, average number of offspring with whom they lived, and whether they had offspring). Thus, individuals in South Korea with poor quality of life need active support and effective programmes and services.

Acknowledgements We thank Textcheck who have checked and corrected the English in our manuscript.

Contributors J-HK, SGL and E-CP designed the study, researched the data, performed the statistical analyses, and wrote the manuscript. JYS. K-HC, $\mathrm{J}$-WC and J-HK contributed to the discussion, and reviewed and edited the manuscript. E-CP is guarantor.

Competing interests None declared.

Provenance and peer review Not commissioned; externally peer reviewed.

Data sharing statement No additional data are available.

Open Access This is an Open Access article distributed in accordance with the Creative Commons Attribution Non Commercial (CC BY-NC 4.0) license, which permits others to distribute, remix, adapt, build upon this work noncommercially, and license their derivative works on different terms, provided the original work is properly cited and the use is non-commercial. See: http:// creativecommons.org/licenses/by-nc/4.0/

\section{REFERENCES}

1. Lim JW. The changing trends in live birth statistics in Korea, 1970 to 2010. Korean J Pediatr 2011;:54:429-35.

2. Won MR, Choi YJ. Are Koreans prepared for the rapid increase of the single-household elderly? Life satisfaction and depression of the single-household elderly in Korea. Scientific World Journal 2013;2013:972194.

3. Khajedaluee M, Assadi R, Dadgar Moghadam M. Health-related quality of life of young addict women in Mashhad, IR Iran. Int $J$ High Risk Behav Addict 2013;2:87-91.

4. World Health Organization Quality of Life Assessment Group. The World Health Organization Quality of Life Assessment (WHOQOL): position paper from the World Health Organization. Soc Sci Med 1995;41:1403.

5. Pinquart M, Sorensen S. Influences of socioeconomic status, social network, and competence on subjective well-being in later life: a meta-analysis. Psychol Aging 2000;15:187-224.

6. Robinson JG, Molzahn AE. Sexuality and quality of life? J Gerontol Nurs 2007:33:19-27; quiz 38-9.

7. Bures RM, Koropeckyj-Cox T, Loree M. Childlessness, parenthood, and depressive symptoms among middle-aged and older adults. $J$ Fam Issues 2009;30:670-87.

8. Chen Y, Hicks A, While AE. Quality of life and related factors: a questionnaire survey of older people living alone in Mainland China. Qual Life Res 2014;23:1593-602.

9. Fratiglioni L, Wang HX, Ericsson K, et al. Influence of social network on occurrence of dementia: a community-based longitudinal study. Lancet 2000;355:1315-19.
10. Lauder W, Sharkey S, Mummery K. A community survey of Ioneliness. J Adv Nurs 2004:46:88-94.

11. Evenson RJ, Simon RW. Clarifying the relationship between parenthood and depression. J Health Soc Behav 2005;46:341-58.

12. Bilgili N, Arpaci F. Quality of life of older adults in Turkey. Arch Gerontol Geriatr 2014;59:415-21.

13. Peel NM, Bartlett HP, Marshall AL. Measuring quality of life in older people: Reliability and validity of WHOQOL-OLD. Australas J Ageing 2007;26:162-7.

14. Ware JE. Conceptualization and measurement of health-related quality of life: comments on an evolving field. Arch Phys Med Rehabil 2003;84(Suppl 2):543-51.

15. Korea Employment Information Service. http://survey.keis.or.kr/

16. Oh B, Cho B, Choi HC, et al. The influence of lower-extremity function in elderly individuals' quality of life (QOL): an analysis of the correlation between SPPB and EQ-5D. Arch Gerontol Geriatr 2014;58:278-82.

17. Kim JH, Park EC. Influence of offspring on quality of life among cancer patients and survivors: results from the Korean longitudinal study of aging (KLoSA), 2008-2011. Asian Pac J Cancer Prev 2014;15:10531-7.

18. Arrandale $\mathrm{VH}$, Koehoorn $\mathrm{M}, \mathrm{MacNab} \mathrm{Y}$, et al. Longitudinal analysis of respiratory symptoms in population studies with a focus on dyspnea in marine transportation workers. Int Arch Occup Environ Health 2009;82:1097-105.

19. Rogers C. The actualizing tendency in relation to 'motives' and to consciousness. In: Jones MR, ed. Nebraska symposium on motivation. Vol. 11. Lincoln: University of Nebraska Press, 1963:1-24.

20. Ryan RM, Deci EL. On happiness and human potentials: a review of research on hedonic and eudaimonic well-being. Annu Rev Psychol 2001;52:141-66.

21. Kim KH, Kim JS, Lee BS, et al. A study about the factors affecting the suicidal thought in Korean elders. J Korean Acad Psychiatr Ment Health Nurs 2010;19:391-9.

22. Choi YM, Lee SJ. Relationship between the quality of life and the social support network for single home-owned old people. J Welfare Aged 2003;22:193-217.

23. Yang $\mathrm{OK}, \mathrm{Ju} \mathrm{SH}$. An exploratory study on the influencing factors to suicidal ideation among single-household elderly population. Ment Health Soc Work 2011;37:321-47.

24. Chappell NL. Correcting cross-cultural stereotypes: aging in Shanghai and Canada. J Cross Cult Gerontol 2003 18:127-47.

25. Friedman LC, Baer PE, Nelson DV, et al. Women with breast cancer: perception of family functioning and adjustment to illness. Psychosom Med 1988;50:529-40.

26. Tramonti F, Barsanti I, Bongioanni $\mathrm{P}$, et al. A permanent emergency: a longitudinal study on families coping with amyotrophic lateral sclerosis. Fam Syst Health 2014;32:271-9.

27. Aarts JW, van Empel IW, Boivin J, et al. Relationship between quality of life and distress in infertility: a validation study of the Dutch FertiQoL. Hum Reprod 2011;26:1112-18.

28. Bolsoy N, Taspinar A, Kavlak O, et al. Differences in quality of life between infertile women and men in Turkey. J Obstet Gynecol Neonatal Nurs 2010;39:191-8.

29. Peronace LA, Boivin J, Schmidt L. Patterns of suffering and social interactions in infertile men: 12 months after unsuccessful treatment. J Psychosom Obstet Gynaecol 2007;28:105-14. 